University of Windsor

Scholarship at UWindsor

1972

\title{
Relativistic corrections to radiative transition probabilities
}

Gordon W. F. Drake

University of Windsor

Follow this and additional works at: https://scholar.uwindsor.ca/physicspub

Part of the Physics Commons

\section{Recommended Citation}

Drake, Gordon W. F.. (1972). Relativistic corrections to radiative transition probabilities. Physical Review A, 5 (5), 1979-1985.

https://scholar.uwindsor.ca/physicspub/112

This Article is brought to you for free and open access by the Department of Physics at Scholarship at UWindsor. It has been accepted for inclusion in Physics Publications by an authorized administrator of Scholarship at UWindsor. For more information, please contact scholarship@uwindsor.ca. 


\title{
Physical REVIEW A GENERAL PHYSICS
}

Third Series, Vol. 5, No. 5

MAY 1972

\section{Relativistic Corrections to Radiative Transition Probabilities*}

\author{
G. W. F. Drake \\ Department of Physics, University of Windsor, Windsor, Ontario, Canada
} (Received 8 April 1971; revised manuscript received 15 October 1971)

\begin{abstract}
The rate of spontaneous emission of radiation by a loosely bound composite system is calculated from quantum electrodynamics correct through terms of relative order $\alpha^{2} Z^{2}$. Recent criticisms by Feinberg and Sucher of the semiclassical method of deriving the higher-order radiation coupling terms are discussed. It is proved that the Breit interaction remains valid in the presence of radiation emission and that the semiclassical and quantum-electrodynamic methods of deriving the higher-order coupling terms always yield the same results to relative order $\alpha^{2} Z^{2}$. We discuss in particular relativistic corrections to allowed and spin-forbidden electric dipole transitions and the $1 s 2 s^{3} S_{1}-1 s^{21} S_{0}$ magnetic dipole transition.
\end{abstract}

\section{INTRODUCTION}

Although a theory in lowest order describing the emission of atomic radiation has existed for many years, ${ }^{1}$ recent laboratory ${ }^{2-4}$ and astrophysical ${ }^{5}$ observations of transitions in which relativistic effects play an important role have stimulated renewed theoretical interest. As examples of such processes, the $1 s 2 p^{3} P_{1}-1 s^{21} S_{0}$ spin-forbidden electric dipole $(E 1)$ transition of the heliumlike ions dominates the allowed $1 s 2 p^{3} P_{1}-1 s 2 s{ }^{3} S_{0}$ transition for nuclear charges $Z \geqslant 5^{6}$ and the $1 s 2 s^{3} S_{1}-1 s^{21} S_{0}$ magnetic dipole $(M 1)$ transition determines the radiative lifetime of the $1 s 2 s{ }^{3} S_{1}$ state for all the heliumlike ions. ${ }^{7}$ In both cases, the lowest-order transition integral vanishes exactly due to spinor or wave-function orthogonality, and it is necessary to evaluate the $O\left(\alpha^{2} Z^{2}\right)$ relativistic corrections ( $\alpha$ is the fine-structure constant $e^{2} / \hbar c$ ).

The semiclassical procedure for evaluating the corrections consists of introducing the vector potential $\overrightarrow{\mathrm{A}}$ for the emitted photon as a prescribed external perturbation into the Dirac-Breit Hamiltonian through the replacement $\overrightarrow{\mathrm{p}} \rightarrow \overrightarrow{\mathrm{p}}-(e / c) \overrightarrow{\mathrm{A}}$, and evaluating the equivalent non relativistic interaction operator of the atom with the radiation field. Feinberg and Sucher ${ }^{8}$ have criticized this procedure on the grounds that third-order diagrams contribute and virtual pair creation in the intermediate state may not be properly accounted for. Although the results of their quantum electrodynamic calculation for the $1 s 2 s^{3} S_{1}-1 s^{21} S_{0} M 1$ transition are in complete agree- ment with the semiclassical derivation of Drake, ${ }^{7}$ the implication is that the results may not agree for other transitions. The claim by Feinberg and Sucher that transverse photon effects are counted twice in Ref. 7 apparently arises from a misinterpretation of the procedure followed.

One could also object that the form of the effective Breit potential describing the electron-electron interaction may alter in the presence of photon emis sion. An assumption in the derivation of the Breit interaction (see Ref. 9) is conservation of the electronic energy before and after electron-electron scattering. Since one of the electrons is in a virtual state as a consequence of photon emission, energy conservation is violated. Of course energy is still conserved for the over-all process of interaction plus radiation. It is often implied that the Breit interaction can be used consistently only to calculate diagonal expectation values, giving the energy shift and fine-structure splitting through terms of relative order $\alpha^{2} Z^{2}$ (see, e.g., Ref. 10, p. 173).

The general problem of the interaction of a loosely bound composite system with an external electromagnetic field has been discussed by Brodsky and Primack $^{11}$ but they did not consider explicitly the emission of radiation. In view of the comments by Feinberg and Sucher ${ }^{8}$ we discuss the emission of radiation by a composite system, starting from basic quantum electrodynamics and including the above relativistic effects. We derive the general form of the relativistic corrections and prove the following two theorems: (i) The form of the Breit 
interaction is unaltered in lowest order by the presence of photon emission. (ii) The $O\left(\alpha^{2} Z^{2}\right)$ corrections to radiative-transition probabilities obtained by the semiclassical method always agree with the quantum-electrodynamic results. The theorem applies whether or not the leading term to the transition integral vanishes. In proving theorem (ii), we do not consider self-interaction radiative corrections, but they are presumably always of relative order $\alpha^{3} \ln \alpha$. Feinberg and Sucher ${ }^{8}$ have shown this to be true for the $1 s 2 s{ }^{3} S-1 s^{2}{ }^{1} S_{0} M 1$ transition.

Theorem (i) implies that one is rigorously jus tified in using the Breit interaction as a first-order perturbation to describe radiative transitions occurring through off-diagonal mixing with intermediate states. The simplest example is the $1 s 2 p$ ${ }^{3} P_{1}-1 s^{21} S_{0}$ transition, which proceeds via the spinorbit mixing of the $2{ }^{3} P_{1}$ state with all the $n^{1} P_{1}$ states. ${ }^{6}$

The calculation of relativistic effects in atomic systems has recently been reviewed by Grant ${ }^{12}$ and by Wadzinski. ${ }^{13}$

\section{BASIC FORMULATION}

We discuss in particular the emission of radiation by a two-electron system. The results can be generalized to an $\mathrm{N}$-electron system by summing the transition operator over all electron pairs. Initially we assume that the electrons are noninteracting and use as a starting point for the calculation the Dirac Hamiltonian for two electrons

$$
H_{D}=\sum_{j=1,2}\left(\vec{\alpha}_{j} \cdot \overrightarrow{\mathrm{p}}_{j}+\beta_{j} m c+e^{2} V_{j}\right)
$$

where $V_{j}=-Z / v_{j} . \quad H_{D}$ has well-defined eigenfunctions $\psi\left(\vec{r}_{1}, \vec{r}_{2}\right)=\psi_{A}\left(\vec{r}_{1}\right) \psi_{B}\left(\vec{r}_{2}\right)$, where $\psi_{A}$ and $\psi_{B}$ are hydrogenic four-component Dirac spinors. The nuclear field is taken as a fixed external potential and the electron-electron interaction via the electromagnetic field treated by perturbation theory.

In lowest order, the emission of radiation of frequency $\omega$ is represented by the Feynman diagram, Fig. 1(a). Using the notation and conventions of Akhiezer and Berestetskii, ${ }^{9}$ the diagram corresponds to the first-order $S$-matrix element

$$
\begin{aligned}
S_{i \rightarrow f}^{(1)} & =-e \int \bar{\psi}_{C}\left(x_{1}\right) \bar{\psi}_{B}\left(x_{2}\right) \hat{A}\left(x_{1}\right) \psi_{A}\left(x_{1}\right) \psi_{B}\left(x_{2}\right) d^{4} x_{1} d^{4} x_{2} \\
& =2 \pi i e \delta\left(E_{i}-E_{f}-\omega\right) \int \psi_{C}^{*}\left(\overrightarrow{\mathrm{r}}_{1}\right) \vec{\alpha}_{1} \cdot \overrightarrow{\mathrm{A}}\left(\overrightarrow{\mathrm{r}}_{1}\right) \psi_{A}\left(\overrightarrow{\mathrm{r}}_{1}\right) d \overrightarrow{\mathrm{r}}_{1} .
\end{aligned}
$$

In the above, $\bar{\psi}=\psi^{*} \beta$, where $\psi^{*}$ is the Hermitian conjugate, $\hat{A}$ denotes the scalar product $A_{\mu} \gamma_{\mu}$ $(\mu=1, \ldots, 4)$ of the vector potential $\vec{A}$, and $x$ represents a four-vector with $\psi(x)=\psi(\overrightarrow{\mathrm{r}}) e^{-i E t}$. We have taken the number of photons present in the initial state to be zero. The diagram corresponding to Fig. 1(a) in which electron 2 radiates is taken into account by multiplying the transition operator by the symmetrizing operator $\delta=1+(1-2)$. Finally, $S_{i \rightarrow f}$ is related to the matrix element $U_{i \rightarrow f}$ of the effective interaction energy of the atom with the radiation field by $S_{i \rightarrow f}=-2 \pi i U_{i \rightarrow f} \delta\left(E_{i}-E_{f}-\omega\right)$. The rate of spontaneous radiation is given by $w=2$ $\times\left|U_{i \rightarrow f}\right|^{2}$.

Since accurate solutions to the nonrelativistic Schrödinger equation are often available, (2) is best evaluated in the low -energy limit $(\alpha Z \ll 1)$ by expanding in powers of $\alpha Z$ and evaluating the lowest-order equivalent nonrelativistic operator. Expressing $\psi$ as

$$
\psi=\left(\begin{array}{l}
\phi \\
\chi
\end{array}\right)
$$

where $\phi$ and $\chi$ are the large and small two-component spinors, the leading terms in the wave-function expansion are

$$
\chi=[(\vec{\sigma} \cdot \vec{p}) / 2 m c] \phi
$$

and

$$
\phi=\left[1-\frac{(\vec{\sigma} \cdot \overrightarrow{\mathrm{p}})^{2}}{8 m^{2} c^{2}}\right] \Phi ;
$$

$\Phi$ satisfies the nonrelativistic Schrödinger equa tion $\left(H_{\mathrm{NR}}-E_{\mathrm{NR}}\right) \Phi=0$. Substituting (3) into (2) and symmetrizing, the lowest-order effective nonrelativistic interaction energy is

$U_{\mathrm{NR}}^{(1)}=-(e / m c) s\left[\overrightarrow{\mathrm{A}}_{1} \cdot \overrightarrow{\mathrm{p}}_{1}+\frac{1}{2} \vec{\sigma}_{1} \cdot \overrightarrow{\mathfrak{H}}_{1}\right]\left[1+O\left(\alpha^{2} Z^{2}\right)\right]$

with the gauge condition $\nabla \cdot \vec{A}=0$. $\overrightarrow{\mathfrak{C}}=\nabla \times \overrightarrow{\mathrm{A}}$ is the magnetic field.

Since atomic states are characterized by angular momentum and parity quantum numbers, it is convenient to expand $\vec{A}$ as a sum over multipoles $\overrightarrow{\mathrm{A}}_{L M}^{(\lambda)}$ of definite angular momentum $L$, component $M$, and parity $(-1)^{L+\lambda+1}$, where $\lambda=0$ designates a magnetic multipole and $\lambda=1$ an electric multipole. For a given multipole, the transition probability between two atomic states with total angular momenta $J_{i}, J_{f}$ and parities $\pi_{i}$ and $\pi_{f}$ vanishes exactly to all orders in $\alpha$ unless the selection rules

$$
\begin{aligned}
& \pi_{i}=\pi_{f}(-1)^{L+\lambda+1}, \quad\left|J_{i}-J_{f}\right| \leqslant L \leqslant J_{i}+J_{f}, \\
& J_{i}=0 \mapsto J_{f}=0,
\end{aligned}
$$

are satisfied (neglecting nuclear spin). If the transition is not forbidden and the transition integral (2) does not vanish in lowest order, then the transition rate $w$ is $O\left[(\omega / c)^{2 L+1} Z^{-2 L}\right]$ for $E L$ transitions and $O\left[(\omega / c)^{2 L+1} c^{-2} Z^{-2 L}\right]$ for $M L$ transitions.

If the transition integral vanishes in lowest order, then it is necessary to evaluate the $O\left(\alpha^{2} Z^{2}\right)$ corrections. These arise from (i) the one-electron relativistic and finite wavelength corrections to $S_{i \rightarrow f}^{(1)}$. The relativistic terms can be obtained for example, by application of the Foldy-Wouthuysen ${ }^{14}$ transformation for time-dependent potentials as 
discussed by Drake. ${ }^{7}$ Finite wavelength effects are included by expanding beyond the leading term the spherical Bessel functions contained in $\vec{A}_{L M}^{(\lambda)} .^{7}$ Since the argument of the Bessel function is $\omega r / c$, only the leading term is ordinarily required in lowest order. (ii) the third-order diagrams dis cussed in Sec. III.

The first- and third-order diagrams generate all the terms in the transition operator describing photon emission through terms of relative order $\alpha^{2} Z^{2}$. Higher-order ladder-type diagrams contribute wave-function corrections to the initial and final states, but do not introduce further terms into the transition operator. The ladder diagrams can be summed to infinity by including the electron-electron interaction in the zero-order Hamiltonian $H_{D}$, as discussed in the Appendix.

\section{EVALUATION OF THIRD-ORDER DIAGRAMS}

Diagrams 1(b) and 1(c) of Fig. 1 represent the combined effects of radiation and electron-electron interaction via the electromagnetic field. The corresponding diagrams in which electron 2 radiates can be taken into account by symmetrizing the transition operator. Each diagram contains a direct and an exchange part, which are included by antisymmetrizing the wave functions. Leaving aside for the moment the symmetrization, the third-or$\operatorname{der} S$-matrix element is

$$
\begin{array}{r}
S_{i \rightarrow f}^{(3)}=-e^{3} \int \bar{\psi}_{C}\left(x_{2}\right) \bar{\psi}_{D}\left(x_{3}\right) K^{(3)}(2,1 ; 3) \psi_{A}\left(x_{1}\right) \psi_{B}\left(x_{3}\right) \\
\times d^{4} x_{1} d^{4} x_{2} d^{4} x_{3},
\end{array}
$$

where

$$
\begin{aligned}
K^{(3)}(2,1 ; 3)= & {\left[\gamma_{\mu} S^{e}(2,1) \hat{A}(1)\right]\left[\gamma_{6}\right] D_{\mu 5}^{c}(2-3) } \\
& +\left[\hat{A}(2) S^{e}(2,1) \gamma_{\mu}\right]\left[\gamma_{0}\right] D_{\mu 5}^{c}(1-3) \\
D_{\mu 5}^{c}(2-3)= & \frac{\delta_{\mu \delta}}{2 \pi i\left|\bar{r}_{2}-\bar{r}_{3}\right|} \int_{-\infty}^{\infty} e^{i \omega^{\prime \prime}\left(t_{2} t_{3}\right)} \\
& \times \cos \left(\omega^{\prime \prime}\left|\overrightarrow{\mathrm{r}}_{2}-\overrightarrow{\mathrm{r}}_{3}\right|\right) d \omega^{\prime \prime}
\end{aligned}
$$

is the photon propagator, and

$$
S^{e}(2,1)=\frac{1}{2 \pi i} \int_{-\infty}^{\infty} d \omega^{\prime} e^{i \omega^{\prime}\left(t_{2}-t_{1}\right)} \sum_{n \pm} \frac{\psi_{n}\left(\overrightarrow{\mathrm{r}}_{2}\right) \psi_{n}\left(\overrightarrow{\mathrm{r}}_{1}\right)}{E_{n}(1-i \epsilon)+\omega^{\prime}}
$$

is a spectral representation of the electron propagator in the external nuclear field. The summation over $n$ includes both positive- and negative-frequency electron states (denoted by $n \pm$ ). In (7a), the first set of square brackets in each product connects $\bar{\psi}_{C}$ with $\psi_{A}$, and the second set connects $\bar{\psi}_{D}$ with $\psi_{B}$. After performing the time integrations in (7), the interaction-energy matrix element becomes

$$
\begin{aligned}
U_{i \rightarrow f}^{(3)}= & e^{3} \sum_{n \pm}\left[\frac{\left\langle\psi_{C} \psi_{D}|B| \psi_{n} \psi_{B}\right\rangle\left\langle\psi_{n} \psi_{B}\left|\vec{\alpha}_{1} \cdot \vec{A}_{1}\right| \psi_{A} \psi_{B}\right\rangle}{E_{n}-E_{A}+\omega}\right. \\
& \left.+\frac{\left\langle\psi_{C} \psi_{D}\left|\vec{\alpha}_{1} \cdot \vec{A}_{1}\right| \psi_{n} \psi_{D}\right\rangle\left\langle\psi_{n} \psi_{D}|B| \psi_{A} \psi_{B}\right\rangle}{E_{n}-E_{c}-\omega}\right],
\end{aligned}
$$

where

$$
B=\frac{\left(1-\vec{\alpha}_{1} \cdot \vec{\alpha}_{2}\right)}{\left|\vec{r}_{1}-\vec{r}_{2}\right|} \cos \left(\omega_{B D}\left|\vec{r}_{1}-\vec{r}_{2}\right|\right) ;
$$

$\omega_{B D}=E_{B}-E_{D}$, and as before, $S_{i \rightarrow f}^{(3)}=-2 \pi i U_{i \rightarrow f}^{(3)} \delta\left(E_{i}\right.$ $\left.-E_{f}-\omega\right)$. Expression (10) bears a close resemblance to the corresponding expressions for two-photon decay, photon-electron scattering, and other twophoton processes except that in this case, one of the photons is absorbed by the second electron. The negative-frequency terms can be interpreted as virtual-pair creation in the intermediate state.

The above expression cannot be reduced to an equivalent nonrelativistic operator because the dependence on $\omega_{B D}$ cannot be eliminated in a general way, but the terms through relative order $\alpha^{2} Z^{2}$ can be extracted by a modification of the argument used by Akhiezer and Berestetskii ${ }^{9}$ in deriving the Breit interaction in the absence of radiation. If the cosine function is expanded, then

$$
B=\frac{\left(1-\vec{\alpha}_{1} \cdot \vec{\alpha}_{2}\right)}{\left|\overrightarrow{\mathrm{r}}_{1}-\overrightarrow{\mathrm{r}}_{2}\right|}-\frac{\left(E_{B}-E_{D}\right)^{2}}{2 c^{2}}\left|\overrightarrow{\mathrm{r}}_{1}-\overrightarrow{\mathrm{r}}_{2}\right|+O\left(c^{-4}\right) \text {. }
$$

In the first term of (10), we replace the second term of (12) by

$$
\begin{aligned}
& -\frac{\left(E_{B}-E_{D}\right)^{2}}{2 c^{2}}\left|\overrightarrow{\mathrm{r}}_{1}-\overrightarrow{\mathrm{r}}_{2}\right| \\
& =\left(E_{n}-E_{C}\right)\left(E_{B}-E_{D}\right)\left|\overrightarrow{\mathrm{r}}_{1}-\overrightarrow{\mathrm{r}}_{2}\right| / 2 c^{2} \\
& \quad \quad+\left(E_{A}-E_{n}-\omega\right)\left(E_{B}-E_{D}\right)\left|\overrightarrow{\mathrm{r}}_{1}-\overrightarrow{\mathrm{r}}_{2}\right| / 2 c^{2} \\
& =\left[H_{1},\left[H_{2},\left|\overrightarrow{\mathrm{r}}_{1}-\overrightarrow{\mathrm{r}}_{2}\right|\right]\right] / 2 c^{2} \\
& \quad-\left(E_{A}-E_{n}-\omega\right)\left[H_{2},\left|\overrightarrow{\mathrm{r}}_{1}-\overrightarrow{\mathrm{r}}_{2}\right|\right] / 2 c^{2} \\
& =\frac{\vec{\alpha}_{1} \cdot \vec{\alpha}_{2}-\left(\vec{\alpha}_{1} \cdot \overrightarrow{\mathrm{n}}\right)\left(\vec{\alpha}_{2} \cdot \overrightarrow{\mathrm{n}}\right)}{2 r_{12}}+\frac{\left(E_{A}-E_{n}-\omega\right)}{2 i c} \vec{\alpha}_{2} \cdot \overrightarrow{\mathrm{n}} \\
& \overrightarrow{\mathrm{n}}=\left(\overrightarrow{\mathrm{r}}_{1}-\overrightarrow{\mathrm{r}}_{2}\right) / r_{12}, \quad r_{12}=\left|\overrightarrow{\mathrm{r}}_{1}-\overrightarrow{\mathrm{r}}_{2}\right|,
\end{aligned}
$$

and in the second term of (10), we replace the second term of (12) by

$$
\begin{aligned}
& -\frac{\left(E_{B}-E_{D}\right)^{2}}{2 c^{2}}\left|\overrightarrow{\mathrm{r}}_{1}-\overrightarrow{\mathrm{r}}_{2}\right| \\
& =\left(E_{A}-E_{n}\right)\left(E_{B}-E_{D}\right)\left|\overrightarrow{\mathrm{r}}_{1}-\overrightarrow{\mathrm{r}}_{2}\right| / 2 c^{2} \\
& \quad+\left(E_{n}-E_{c}-\omega\right)\left(E_{B}-E_{D}\right)\left|\overrightarrow{\mathrm{r}}_{1}-\overrightarrow{\mathrm{r}}_{2}\right| / 2 c^{2} \\
& =\frac{\vec{\alpha}_{1} \cdot \vec{\alpha}_{2}-\left(\vec{\alpha}_{1} \cdot \overrightarrow{\mathrm{n}}\right)\left(\vec{\alpha}_{2} \cdot \overrightarrow{\mathrm{n}}\right)}{2 r_{12}}+\frac{\left(E_{n}-E_{C}-\omega\right)}{2 i c} \vec{\alpha}_{2} \cdot \overrightarrow{\mathrm{n}}
\end{aligned}
$$

The energy terms multiplying $\vec{\alpha}_{2} \cdot \vec{n}$ in (13) and (14) cancel with their respective energy denominators in 
(10), and the sum over $n \pm$ can be carried out exactly by closure. Collecting the results and defining

$$
\begin{aligned}
B_{V} & =e^{2} / r_{12}-\left(e^{2} / 2 r_{12}\right)\left[\vec{\alpha}_{1} \cdot \vec{\alpha}_{2}+\left(\vec{\alpha}_{1} \cdot \overrightarrow{\mathrm{n}}\right)\left(\vec{\alpha}_{2} \cdot \overrightarrow{\mathrm{n}}\right)\right] \\
& =V_{12}+B,
\end{aligned}
$$

where $B$ is the Breit interaction, the expression for $U_{i \rightarrow f}^{(3)}$ becomes

$$
\begin{aligned}
U_{i \rightarrow f}^{(3)}= & e\left[\sum _ { n \pm } \left(\frac{\left\langle\psi_{C} \psi_{D}\left|B_{V}\right| \psi_{n} \psi_{B}\right\rangle\left\langle\psi_{n} \psi_{B}\left|\vec{\alpha}_{1} \cdot \vec{A}_{1}\right| \psi_{A} \psi_{B}\right\rangle}{E_{n}-E_{A}+\omega}\right.\right. \\
& \left.+\frac{\left\langle\psi_{C} \psi_{D}\left|\vec{\alpha}_{1} \cdot \vec{A}_{1}\right| \psi_{n} \psi_{D}\right\rangle\left\langle\psi_{n} \psi_{D}\left|B_{V}\right| \psi_{A} \psi_{B}\right\rangle}{E_{n}-E_{C}-\omega}\right) \\
& \left.+(1 / i c)\left\langle\psi_{C} \psi_{D}\left|\left[\vec{\alpha}_{1} \cdot \vec{A}_{1}, \vec{\alpha}_{2} \cdot \vec{n}\right]\right| \psi_{A} \psi_{B}\right\rangle\right]
\end{aligned}
$$

The last term of (16) is the additional contribution due to nonconservation of energy in the virtualelectron state. The extra term vanishes provided that $\vec{A}_{1}$ and $\vec{n}$ commute. No approximation has been made other than truncating the retardation expansion (12). Since $B$ in (15) is the Breit interaction, theorem (i) is established.

The next step in the reduction of (16) is the evalua tion of the sum over negative frequencies, which we denote by $U_{i \rightarrow f}^{(3)}$. In the low-energy limit, we can assume $\omega \ll m c^{2},\left|E_{A}-\omega\right| \ll m c^{2}$, and $\left|E_{C}-\omega\right|$ $\ll m c^{2}$. Since $E_{n-} \sim-m c^{2}$, the denominators in (16) can be replaced by $-2 m c^{2}$ to the required degree of accuracy. If we introduce into $U_{i \rightarrow f}^{(3)-}$ the negativefrequency projection operator

$$
\Lambda_{-}=\frac{m c^{2}-H_{1}}{m c^{2}-E_{n_{-}}} \simeq \frac{1}{2}\left[1-\beta_{1}-\vec{\alpha}_{1} \cdot \vec{p}_{1} /(m c)\right]
$$

with the properties $\Lambda_{-} \psi_{n_{-}}=\psi_{n-}, \Lambda_{-} \psi_{n^{+}}=0$, then the sum over $n$ - can be extended to a sum over $n \pm$ without altering the value of $U_{i \rightarrow f}^{(3)-}$. The sum over $n \pm$ can now be evaluated by closure with the final result

$$
\begin{aligned}
U_{i \rightarrow f}^{(3)-} \simeq\left[-e /\left(2 m c^{2}\right)\right]\left\langle\psi_{c}\right. & \psi_{D} \mid B_{V} \Lambda_{-} \vec{\alpha}_{1} \cdot \overrightarrow{\mathrm{A}}_{1} \\
& +\vec{\alpha}_{1} \cdot \overrightarrow{\mathrm{A}}_{1} \Lambda_{-} B_{V}\left|\psi_{A} \psi_{B}\right\rangle .
\end{aligned}
$$

The above is in harmony with the corresponding expression obtained by Feinberg and Sucher ${ }^{8}$ in their discussion of $M 1$ transitions. Introducing approximation (3) for the small components of the wave functions, the expression becomes

$$
\begin{aligned}
U_{i \rightarrow f}^{(3)-} \simeq & {\left[-e^{3} /\left(4 m^{2} c^{3}\right)\right]\left\langle\phi_{C} \phi_{D}\right|\left\{\left[\left(\vec{\sigma}_{1} \cdot \overrightarrow{\mathrm{p}}_{1}\right), v_{12}^{-1}\left(\vec{\sigma}_{1} \cdot \overrightarrow{\mathrm{A}}_{1}\right)\right]_{+}\right.} \\
& -\left[\left(\vec{\sigma}_{2} \cdot \overrightarrow{\mathrm{p}}_{2}\right),\left[\left(\vec{\sigma}_{1} \cdot \overrightarrow{\mathrm{A}}_{1}\right), B_{\sigma}\right]_{+}\right]_{+} \\
& \left.-\left(\vec{\sigma}_{1} \cdot \overrightarrow{\mathrm{A}}_{1}\right)\left[\left(\vec{\sigma}_{1} \cdot \overrightarrow{\mathrm{p}}_{1}\right), v_{12}^{-1}\right]_{+}\right\}\left|\phi_{A} \phi_{B}\right\rangle, \quad(19)
\end{aligned}
$$

where

$$
B_{\sigma}=\left[1 /\left(2 r_{12}\right)\right]\left[\vec{\sigma}_{1} \cdot \vec{\sigma}_{2}+\left(\vec{\sigma}_{1} \cdot \vec{n}\right)\left(\vec{\sigma}_{2} \cdot \vec{n}\right)\right],
$$

and $[,]_{+}$denotes the anticommutator. In the effective nonrelativistic form of the Breit interaction $\left(B_{\mathrm{NR}}\right)$, the orbit-orbit, spin-spin, and that part of the spin-other-orbit terms arising from $B_{\sigma}$ are obtained by calculating the nonrelativistic equivalent to $\left[-e^{2} /\left(4 m^{2} c^{2}\right)\right]\left[\left(\vec{\sigma}_{2} \cdot \overrightarrow{\mathrm{p}}_{2}\right),\left[\left(\vec{\sigma}_{1} \cdot \overrightarrow{\mathrm{p}}_{1}\right), B_{\sigma}\right]_{+}\right]_{+}$, with allowance for the $\delta\left(\overrightarrow{\mathrm{r}}_{12}\right)$ singularity in the spin-spin term (see, e.g., Ref. 9, pp. 517-521). Since the form of the nonrelativistic equivalent operator is invariant under the replacement of $\overrightarrow{\mathrm{p}}_{j}$ by $\vec{\pi}_{j}=\overrightarrow{\mathrm{p}}_{j}$ $-(e / c) \overrightarrow{\mathrm{A}}_{j}$, provided $\nabla \cdot \overrightarrow{\mathrm{A}}=0$, the nonrelativistic equivalent to the symmetrized terms $\left[e^{3} /\left(4 m^{2} c^{3}\right)\right]$ $\times S\left[\left(\vec{\sigma}_{2} \cdot \overrightarrow{\mathrm{p}}_{2}\right),\left[\left(\vec{\sigma}_{1} \cdot \overrightarrow{\mathrm{A}}_{1}\right), B_{\sigma}\right]_{+}\right]_{+}$of $(19)$ can be obtained by the semiclassical procedure of replacing $\vec{p}$ by $\overrightarrow{\mathrm{p}}-(e / c) \overrightarrow{\mathrm{A}}$ in the above parts of $B_{\mathrm{NR}}$. When the other two terms of (19) are added, the symmetrized interaction-energy operator is

$$
\begin{aligned}
U_{\mathrm{NR}}^{(3)-}=\left[e^{3} /\left(2 m^{2} c^{3}\right] s\left[-r_{12}^{-3}\left(\vec{\sigma}_{1}+\vec{\sigma}_{2}\right) \cdot \overrightarrow{\mathrm{r}}_{12} \times \overrightarrow{\mathrm{A}}_{1}\right.\right. \\
\left.+r_{12}^{-1} \overrightarrow{\mathrm{A}}_{1} \cdot \overrightarrow{\mathrm{p}}_{2}+r_{12}^{-3}\left(\overrightarrow{\mathrm{r}}_{12} \cdot \overrightarrow{\mathrm{A}}_{1}\right)\left(\overrightarrow{\mathrm{r}}_{12} \cdot \overrightarrow{\mathrm{p}}_{2}\right)\right] .
\end{aligned}
$$

The above contains all the corrections of relative order $\alpha^{2} Z^{2}$ which depend explicitly on the $r_{12}$ coordinate. The result is valid whether or not the leading term vanishes and applies to all multipole transitions since no assumption is made about the nature of $\overrightarrow{\mathrm{A}}_{1}$, other than the Coulomb-gauge condition $\nabla \cdot \vec{A}=0$. The corresponding part of the interaction operator derived semiclassically ${ }^{7}$ is exactly the same as (20) for arbitrary potentials, thus proving theorem (ii).

As mentioned previously, the one-electron relativistic corrections to $U_{i \rightarrow f}^{(1)}$ can be calculated rigorously by application of the Foldy-Wouthuysen transformation. Using the results derived in Ref. 7 , the total "direct" part of the interaction operator is

$$
\begin{aligned}
U_{\mathrm{NR}}^{\mathrm{dir}}= & U_{\mathrm{NR}}^{(1)}+U_{\mathrm{NR}}^{(3)-} \\
= & {[-e /(m c)] \mathrm{s}\left[\overrightarrow{\mathrm{A}}_{1} \cdot \overrightarrow{\mathrm{p}}_{1}+\frac{1}{2} \vec{\sigma}_{1} \cdot \overrightarrow{\mathcal{H}}_{1}\right] } \\
& +\left[e^{3} /\left(2 m^{2} c^{3}\right)\right] s\left\{-\vec{\sigma}_{1} \cdot \nabla_{1} V_{1} \times \overrightarrow{\mathrm{A}}_{1}\right. \\
& +\frac{1}{2}\left[p_{1}^{2}, \overrightarrow{\mathrm{A}}_{1} \cdot \overrightarrow{\mathrm{p}}_{1}+\frac{1}{2} \vec{\sigma}_{1} \cdot \overrightarrow{\mathfrak{H}}_{1}\right]+ \\
& \left.+\frac{1}{8}\left[p_{1}^{2}, \vec{\sigma}_{1} \cdot \overrightarrow{\mathfrak{H}}_{1}+2 i \overrightarrow{\mathrm{A}}_{1} \cdot \vec{\sigma}_{1} \times \overrightarrow{\mathrm{p}}_{1}\right]-\right\}+U_{\mathrm{NR}}^{(3)-}
\end{aligned}
$$

correct through terms of relative order $\alpha^{2} Z^{2}$. To obtain the above, the factor of $\omega$ in Eq. (28) of Ref. 7 is dropped, and the operators it multiplies are replaced by the commutators of the operators with the zero-order nonrelativistic Hamiltonian $H_{\mathrm{NR}}^{0}=\sum_{j}\left(-\frac{1}{2} \nabla_{j}^{2}+V_{j}\right)$. Terms arising from the $e^{2} / r_{12}$ interaction are already included in Eq. (20).

The remaining part of (16) containing the sum over positive-frequency states is denoted by $U_{i \rightarrow f}^{(3)+}$. To the desired degree of accuracy, it can be written in terms of nonrelativistic two-electron eigenfunctions $\Phi$ of $H_{\mathrm{NR}}$ as

$$
U_{i \rightarrow f}^{(3)+} \simeq \sum_{n+}\left[\frac{\left\langle\Phi_{f}\left|B_{\mathrm{NR}}\right| \Phi_{n}\right\rangle\left\langle\Phi_{n}\left|U_{\mathrm{NR}}^{(1)}\right| \Phi_{i}\right\rangle}{E_{f}-E_{n}}\right.
$$




$$
\left.+\frac{\left\langle\Phi_{f}\left|U_{\mathrm{NR}}^{(1)}\right| \Phi_{n}\right\rangle\left\langle\Phi_{n}\left|B_{\mathrm{NR}}\right| \Phi_{i}\right\rangle}{E_{i}-E_{n}}\right],
$$

where $E_{i}$ and $E_{f}$ are the exact nonrelativistic twoelectron initial- and final-state energies, and $U_{\mathrm{NR}}^{(1)}$ is given by (5). The $e^{2} / r_{12}$ term is no longer treated as a perturbation, but is included in $H_{\mathrm{NR}}$ by summing the ladder diagrams, as discussed in the Appendix. $U_{i \rightarrow f}^{(3)+}$ then represents first-order wavefunction corrections to the initial and final states due to the Breit interaction $B_{\mathrm{NR}}$, and is to be added to the matrix element $\left\langle\Phi_{f}\left|U_{\mathrm{NR}}^{\mathrm{ddr}}\right| \Phi_{i}\right\rangle$ to obtain the total transition integral $U_{i \rightarrow f}$ correct through terms of relative order $\alpha^{2} Z^{2}$. In the nonrelativistic limit, the states $\Phi_{i}$ and $\Phi_{f}$ are strictly $L S$ coupled. Higher-order effects such as spin-orbit mixing are accounted for by (22).

The results can be expressed more compactly by introducing the first-order corrected functions

$$
\Phi_{i}^{\prime}=\Phi_{i}+\sum_{n \neq i} \frac{\left\langle\Phi_{i}\left|B_{\mathrm{NR}}\right| \Phi_{n}\right\rangle \Phi_{n}}{E_{i}-E_{n}},
$$

and similarly for $\Phi_{f}^{\prime}$. $\Phi^{\prime}$ is an eigenfunction of $H_{\mathrm{NR}}^{\prime}=H_{\mathrm{NR}}+B_{\mathrm{NR}}$ through terms of relative order $\alpha^{2} Z^{2}$. $U_{i \rightarrow f}$ can then be written

$$
U_{i \rightarrow f}=\left\langle\Phi_{f}^{\prime}\left|U_{\mathrm{NR}}^{\mathrm{dir}}\right| \Phi_{i}^{\prime}\right\rangle
$$

provided that only terms up to relative order $\alpha^{2} Z^{2}$ are retained. For $M 1$ transitions, $U_{\mathrm{NR}}^{\mathrm{dir}}$ reduces to the expression obtained in Ref. 7.

\section{APPLICATION TO ELECTRIC DIPOLE TRANSITIONS}

Spin-forbidden $E 1$ transitions have been extensively discussed from a less fundamental point of view and reviewed by Goodman and Laurenzi. ${ }^{15}$ The present analysis is more general in that it applies also to allowed transitions and considers the explicit two-electron effects given by $U^{(3)-}$ [Eq. (20)]. Since these terms are symmetric in $\vec{\sigma}_{1}$ and $\vec{\sigma}_{2}$, they do not contribute to transitions involving a spin change. For the case $\lambda=1, L=1$, and $M=0, A_{L M}^{(\lambda)}$ is given $\mathrm{by}^{9}$

$$
\overrightarrow{\mathrm{A}}_{10}^{(1)}=(2 \omega / 3 c)^{1 / 2}\left[\hat{k}_{z}+O\left(\omega^{2} r^{2} / c^{2}\right)\right],
$$

and $\overrightarrow{\mathfrak{H}}_{1 M}^{(1)} \simeq-\left(\omega^{2} / 2 c^{2}\right) \overrightarrow{\mathrm{r}} \times \overrightarrow{\mathrm{A}}_{1 M}^{(1)}$. In the above, $\hat{k}_{k}$ is a unit vector in the $z$ direction. The term $-\frac{1}{2} \vec{\sigma} \cdot \overrightarrow{\mathcal{H}}$ in (21) then becomes $\left(\omega^{2} / 4 c^{2}\right) \vec{\sigma} \cdot \overrightarrow{\mathrm{r}} \times \overrightarrow{\mathrm{A}}_{1 M}^{(1)}$. If we replace the $\omega^{2} \overrightarrow{\mathrm{r}}$ factor by the double commutator $\left[H_{\mathrm{NR}},\left[H_{\mathrm{NR}}, \overrightarrow{\mathrm{r}}_{1}\right]\right]=\left(e^{2} / m\right)\left(\nabla V_{1}-\overrightarrow{\mathrm{r}}_{12} / r_{12}^{3}\right)$ and omit the spin-symmetric and spin-independent terms, then $U_{\mathrm{NR}}^{\mathrm{dir}}$ reduces to

$$
\begin{aligned}
U_{\mathrm{NR}}^{\mathrm{dir}}= & -(e / m c) s\left(\overrightarrow{\mathrm{A}}_{1} \cdot \overrightarrow{\mathrm{p}}_{1}\right)-\left[e^{3} /\left(2 m^{2} c^{3}\right)\right] \\
& \times s\left[\frac{1}{2} \vec{\sigma}_{1} \cdot \nabla_{1} V_{1} \times \overrightarrow{\mathrm{A}}_{1}+r_{12}^{-3} \vec{\sigma}_{1} \cdot \overrightarrow{\mathrm{r}}_{12} \times \overrightarrow{\mathrm{A}}_{1}\right]
\end{aligned}
$$

for spin-forbidden $E 1$ transitions. Since

$$
\begin{aligned}
-i\left(E_{i}\right. & \left.-E_{f}\right)(2 \omega / 3 c)^{1 / 2}\left\langle\Phi_{f}^{\prime}\left|\hat{k}_{z} \cdot\left(\overrightarrow{\mathrm{r}}_{1}+\overrightarrow{\mathrm{r}}_{2}\right)\right| \Phi_{i}^{\prime}\right\rangle \\
& =-i(2 \omega / 3 c)^{1 / 2}\left\langle\Phi_{f}^{\prime} \mid\left[\hat{k}_{z} \cdot\left(\overrightarrow{\mathrm{r}}_{1}+\overrightarrow{\mathrm{r}}_{2}\right), H_{\mathrm{NR}}^{\prime}\right] \Phi_{i}^{\prime}\right\rangle \\
& =\left\langle\Phi_{f}^{\prime}\left|U_{\mathrm{NR}}^{\mathrm{dir}}\right| \Phi_{i}^{\prime}\right\rangle,
\end{aligned}
$$

the transition integrals are correctly calculated in the "dipole-length" form as shown also by Goodman and Laurenzi. ${ }^{15}$ The above argument can be extended to higher electric multipoles since $\overrightarrow{\mathrm{A}}_{L M}^{(1)}$ and $H_{\mathrm{NR}}$ commute to the necessary degree of accuracy.

We consider also allowed $E 1$ transitions in which the principle quantum number does not change, such as the $1 s 2 p{ }^{3} P-1 s 2 s{ }^{3} S$ transition of the heliumlike ions. Since the nonrelativistic transition frequency increases only as $Z$ while the relativistic corrections increase as $Z^{4}$, the corrections may become large, even for small $Z$. If we assume that the "long-wavelength" approximation is still valid ( $\omega r_{0} / c \ll 1$, where $r_{0}$ is the atomic radius), then the major part of the relativistic correction to the transition rate is obtained by replacing the nonrelativis tic energy difference by the relativistically corrected (or experimental) one. Since the equivalence of the length and velocity forms of the transition integral holds also for Dirac wave functions, i. e.,

$$
\int \psi_{2}^{*} \vec{\alpha} \cdot \hat{k} \psi_{1} d \overrightarrow{\mathrm{r}}=-i\left(E_{i}-E_{f}\right) \int \psi_{2}^{*} \overrightarrow{\mathrm{r}} \cdot \hat{k} \psi_{1} d \overrightarrow{\mathrm{r}},
$$

a large part of the relativistic correction to the velocity form of the transition integral on the lefthand side of (28) is expressed simply as an energy difference multiplying the length form on the righthand side. It is physically reasonable to suppose that the integral on the right-hand side is less sensitive to relativistic corrections because $\vec{r}$ weights the parts of the wave functions far from the nucleus. Thus, the length form is the correct one to use in applying the above procedure. For the $1 s 2 p^{3} P_{2}$ $-1 s 2 s{ }^{3} S_{1}$ transition of heliumlike Ar XVII $(Z=18)$, the relativistic energy correction increases the transition rate by nearly a factor of $2 .^{16}$

\section{DISCUSSION}

We have obtained from first principles all of the radiation coupling terms correct to relative order $\alpha^{2} Z^{2}$ and have shown that the semiclassical and quantum electrodynamic derivations will always give exactly the same results to this order. Thus, the semiclassical derivation of the $M 1$ transition probability presented in Ref. 7 is as well founded as quantum electrodynamics itself. It is interesting that the summation over negative-energy states (virtual-pair creation) leads to exactly the same coupling terms as those obtained by the semiclassical procedure applied directly to the Breit interaction.

The development of high-energy beam-foil experiments has made possible the accurate measurement of radiative lifetimes in highly ionized sys tems such as Ar XVII. For the $1 s 2 s^{3} S_{1}-1 s^{21} S_{0} M 1$ transition of this system, Schmieder and Marrus ${ }^{4}$ have measured a lifetime of $172 \pm 30 \mathrm{nsec}$, which is slightly below the theoretical value 212 nsec. ${ }^{7}$ The $10-20 \%$ difference, if real, can perhaps be accounted 

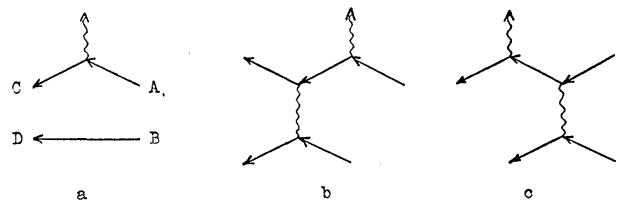

FIG. 1. First- and third-order contributions to the spontaneous emission of radiation by a two-electron system. The solid lines represent electrons and the wavy lines represent photons. Each diagram has a partner in which the other electron radiates.

for by the next correction of relative order $\alpha Z$ to the two-electron terms $U^{(3)-}$. In addition, there is a small one-electron Lamb-shift-type correction of relative order $\alpha \ln \alpha .^{8}, 17$ The present analysis lays a firm foundation for the evaluation of these terms.

\section{ACKNOWLEDGMENT}

Part of this work was done while the author was visiting at the Harvard College Observatory. The hospitality of Professor A. Dalgarno and the Harvard College Observatory is gratefully acknowledged.

\section{APPENDIX}

In deriving the $O\left(\alpha^{2} Z^{2}\right)$ corrections to the photon transition operator, we have treated the entive interelectronic interaction, including the $V=e^{2} / r_{12}$ term, as a perturbation. However, the eigenfunctions of $H_{D}$ [cf, Eq. (1)] form a complete set, and antisymmetrized linear combinations can be formed which are eigenfunctions of the total atomic Hamiltonian $H_{\text {tot }}=H_{D^{+}} V+B$, up to terms of $O\left(\alpha^{2} Z^{2}\right)$. It then seems likely that the same transition operator,

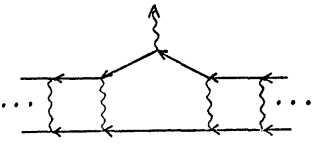

FIG. 2. General ladder-diagram contribution to the spontaneous emission of radiation by a two-electron system.

when expressed in a form independent of the initialand final-state frequencies, applies also to transitions between states described by eigenfunctions of $H_{\text {tot }}$, provided that corrections of higher order than $\alpha^{2} Z^{2}$ are discarded.

The above proposition may be proved as follows. Assume throughout that terms of higher order than $\alpha^{2} Z^{2}$ are discarded. The diagrams shown in Fig. 1 are the leading terms in an infinite sequence of ladder-type diagrams of the general form shown in Fig. 2 with all possible time orderings. The sum of all the $2 n+1$ order diagrams yields exactly the same photon transition operator in each case, in addition to all the $n$ th-order wave-function corrections arising from the perturbation $V+B$. The sum of all the ladder diagrams includes the $e^{2} / r_{12}$ term to infinite order as a $Z^{-1}$ expansion-perturbation series and the $\alpha^{2} Z^{2}$ corrections to first order. Diagrams with crossed quantum lines introduce further corrections of relative order $\alpha^{3}$ and are not included.

As a specific example, consider the fifth-order diagrams shown in Fig. 3. The contribution to $S_{i \rightarrow f}^{(5)}$ is

$$
\begin{aligned}
S_{i \rightarrow f}^{(5), f^{\text {adde }}=}= & -e^{5} \int \bar{\psi}_{C}\left(x_{4}\right) \bar{\psi}_{D}\left(x_{5}\right) K^{(5)}(4,2,1 ; 5,3) \\
& \times \psi_{A}\left(x_{1}\right) \psi_{B}\left(x_{3}\right) d x_{1}, \cdots, d x_{5},
\end{aligned}
$$

where

$$
\begin{aligned}
K^{(5)}(4,2,1 ; 5,3)=\left[\gamma_{\nu} S^{e}(4,2)\right. & \left.\gamma_{\mu} S^{e}(2,1) \hat{A}(1)\right]\left[\gamma_{\lambda} S^{e}(5,3) \gamma_{\delta}\right] D_{\nu \lambda}^{c}(4-5) D_{\mu \delta}^{c}(2-3) \\
+ & {\left[\gamma_{\nu} S^{e}(4,2) \hat{A}(2) S^{e}(2,1) \gamma_{\mu}\right]\left[\gamma_{\lambda} S^{e}(5,3) \gamma_{6}\right] D_{\nu \lambda}^{c}(4-5) D_{\mu \delta}^{c}(1-3) } \\
& +\left[\hat{A}(4) S^{e}(4,2) \gamma_{\nu} S^{e}(2,1) \gamma_{\mu}\right]\left[\gamma_{\lambda} S^{e}(5,3) \gamma_{5}\right] D_{\nu \lambda}^{c}(2-5) D_{\mu \delta}^{c}(1-3) .
\end{aligned}
$$

Equations (8) and (9) are substituted for $S^{e}$ and $D^{c}$, and the integrations over $t_{1}, \cdots, t_{5}$ carried out. A further integration over the virtual-electron and -phononfrequencies is performed by evaluating the integrand at the poles of $S^{e}$ and using the residue theorem. After some algebra and several operator replacements similar to (13) and (14), $U_{i \rightarrow f}^{(5), \text { ladder }}$ becomes

$$
\begin{array}{r}
U_{i \rightarrow f}^{(5), 1 \text { adder }}=-e s\left\langle C D\left|(V+B) G_{0}^{C D}(V+B) G_{0}^{C D} \vec{\alpha}_{1} \cdot \overrightarrow{\mathrm{A}}_{1}+(V+B) G_{0}^{C D} \vec{\alpha}_{1} \cdot \overrightarrow{\mathrm{A}}_{1} G_{0}^{A B}(V+B)+\vec{\alpha}_{1} \cdot \overrightarrow{\mathrm{A}}_{1} G_{0}^{A B}(V+B) G_{0}^{A B}(V+B)\right| A B\right\rangle \\
+[e s /(i c)]\left\langle C D\left|(V+B) G_{0}^{C D}\left[\vec{\alpha}_{2} \cdot \overrightarrow{\mathrm{n}}, \vec{\alpha}_{1} \cdot \overrightarrow{\mathrm{A}}_{1}\right]+\left[\vec{\alpha}_{2} \cdot \overrightarrow{\mathrm{n}}, \vec{\alpha}_{1} \cdot \overrightarrow{\mathrm{A}}_{1}\right] G_{0}^{A B}(V+B)\right| A B\right\rangle, \quad(\mathrm{A} 3)
\end{array}
$$

where

$$
G_{0}^{A B}=\sum_{n_{1}, n_{2}} \frac{\left|n_{1} n_{2}\right\rangle\left\langle n_{1} n_{2}\right|}{E_{A}+E_{B}-E_{n_{1}}-E_{n_{2}}},
$$

and similarly for $G_{0}^{C D}$. Equation (A4) is a spectral representation of the resolvent operator $\left(E_{A}+E_{B}-H_{D}\right)^{-1}$. The summations over negative-energy states $n_{1}$ or $n_{2}$ occurring in matrix elements of the form

$$
\frac{\left\langle C D|\ldots(V+B)| n_{1} n_{3}\right\rangle\left\langle n_{1} n_{3}\left|\vec{\alpha}_{1} \cdot \overrightarrow{\mathrm{A}}_{1}\right| n_{2} n_{3}\right\rangle\left\langle n_{2} n_{3}|(V+B) \ldots| A B\right\rangle}{\left(E_{C}+E_{D}-E_{n_{1}}-E_{n_{3}}\right)\left(E_{A}+E_{B}-E_{n 1}-E_{n 3}\right)}
$$



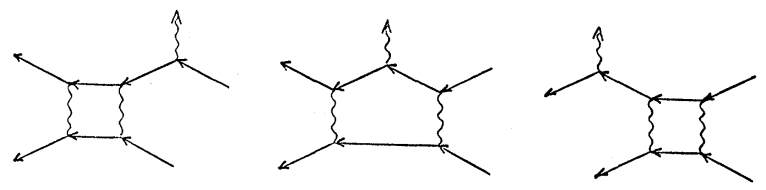

FIG. 3. Fifth-order ladder-diagram contributions to the spontaneous emission of radiation by a two-electron system.

can be carried out by introducing the negative-frequency projection operator $\Lambda_{\text {_ }}$ and using closure in the same way as described in Sec. III. The result is again the operator $U^{(3)-}$ given by (20). Equation (A3) can then be written

$$
\begin{array}{rl}
U_{i \rightarrow f}^{(5), 1 \text { adder }}=-e & S \sum_{m=0}^{2}{ }_{m}\left\langle C D\left|\Lambda_{+} \vec{\alpha}_{1} \cdot \overrightarrow{\mathrm{A}}_{1} \Lambda_{+}\right| A B\right\rangle_{2-m} \\
& +\sum_{m=0}^{1}{ }_{m}\left\langle C D\left|U^{(3)-}\right| A B\right\rangle_{1-m},
\end{array}
$$

where $|A B\rangle_{m}=\left[G_{0}^{A B}(V+B)\right]^{m}|A B\rangle$. For brevity, we have dropped the commutator term in (A3) since it will not normally contribute, but it can be carried through by adding it to $U^{(3) \text { - }}$.

Since the ladder diagrams beyond fifth order just contain further ladder extensions to those shown in Fig. 3, they serve to extend the perturbation expansions of the initial and final states in powers of $Z^{-1}$ without contributing new terms to the transition operator. The infinite sum of all ladder diagrams is then

$$
U_{i \rightarrow f}^{1 \text { adder }}=e \sum_{m, n}^{\infty}{ }_{m}\left\langle C D\left|-\delta \Lambda_{+} \vec{\alpha}_{1} \cdot \overrightarrow{\mathrm{A}}_{1} \Lambda_{+}+U^{(3)-}\right| A B\right\rangle_{n} .
$$

The Breit interaction $B$ is to be included once, in all possible ways, in evaluating the first term of (A6) and not at all in evaluating the second term. If we neglect for the moment the initial- and finalstate level shifts, then the quantity $\sum_{n=0}^{\infty}\left[G_{0}^{A B}(V+B)\right]^{n}$ $|A B\rangle$ is just the perturbation expansion for the eigenfunctions of $H_{\text {tot }}$ with $V+B$ acting as the perturbation. $B$ is of course retained only to first order in evaluating the eigenfunctions of $H_{\text {tot }}$.

The electron-electron interaction is a mutual selfenergy effect and the resulting level shifts to the bound initial and final states can be included formally by introducing the level-shift operator $\Delta$ defined by

$$
\Delta=\sum_{n_{1}, n_{2}}\left|n_{1} n_{2}\right\rangle \Delta_{n_{1} n_{2}}\left\langle n_{1} n_{2}\right|
$$

where

$$
\begin{aligned}
& \Delta_{n_{1} n_{2}}=E_{n_{1} n_{2}}-\left(E_{n_{1}}+E_{n_{2}}\right), \\
& H_{D}\left|n_{1} n_{2}\right\rangle=\left(E_{n_{1}}+E_{n_{2}}\right)\left|n_{1} n_{2}\right\rangle,
\end{aligned}
$$

and $E_{n_{1} n_{2}}$ is the corresponding eigenvalue of $H_{\text {tot }}$. $(V+B)$ is then replaced throughout by $(V+B-\Delta)$, and $H_{D}$ is replaced by $H_{D}+\Delta$. The rest of the analysis is unchanged except that additional terms appear in (A3) and the subsequent equations as a result of the perturbation corrections to the initialand final-state energies. Also the singular terms, such as $E_{n_{1}}=E_{A}, E_{n_{2}}=E_{B}$ in (A6), are subtracted out of the perturbation expansions.

Thus, to relative orders $\alpha^{2} Z^{2}$, one is fully justified in including the $e^{2} / r_{12}$ term in the zero-order Hamiltonian and evaluating matrix elements of the transition operator [or its equivalent nonrelativistic form as in Eq. (24)] between eigenfunctions which take into account the $e^{2} / \gamma_{12}$ interaction exactly and $B$ to first order.
${ }^{*}$ Research supported by the National Research Council of Canada.

${ }^{1} \mathrm{~W}$. Heitler, The Quantum Theory of Radiation (Oxford U. P., London, 1954).

${ }^{2}$ I. A. Sellin, B. Donnally, and C. Y. Fan, Phys. Rev. Letters 21, 717 (1968).

${ }^{3}$ I. A. Sellin, M. Brown, W. W. Smith, and B. Donnally, Phys. Rev. A 2 , 1189 (1970).

${ }^{4}$ R. W. Schmieder and R. Marrus, Phys. Rev. Letters 25, $1245 \cdot(1970)$; R. Marrus and R. W. Schmieder, ibid. $\underline{25}, 1689$ (1970).

${ }^{5}$ A. H. Gabriel and C. Jordan, Monthly Notices Roy. Astron. Soc. 145, 241 (1969); and Nature 221, 947 (1969).

${ }^{6}$ G. W. F. Drake and A. Dalgarno, Astrophys. J. 157, 459 (1969).

${ }^{7}$ G. W. F. Drake, Phys. Rev. A $\underline{3}, 908$ (1971).

${ }^{8}$ G. Feinberg and J. Sucher, Phys. Rev. Letters 26, 681 (1971).
${ }^{9}$ A. I. Akhiezer and V. B. Berestetskii, Quantum Electrodynamics (Interscience, New York, 1965).

${ }^{10} \mathrm{H}$. A. Bethe and E. E. Salpeter, Quantum Mechanics of One- and Two-Electron Atoms (Springer-Verlag, Berlin, 1967).

${ }^{11}$ S. J. Brodsky and J. R. Primack, Ann. Phys. (N. Y.) 52, 315 (1969); see also R. A. Krajcik and L. L. Foldy, Phys. Rev. Letters 24, 545 (1970).

${ }^{12}$ I. P. Grant, Advan. Phys. 19, 747 (1970)

${ }^{13}$ H. T. Wadzinski, J. Phys. (Paris) 31, C4-213 (1970).

${ }^{14}$ L. L. Foldy and S. A Wouthuysen, Phys. Rev. $\underline{78}$, 29 (1950).

${ }^{15}$ L. Goodman and B. J. Laurenzi, Advan. Quantum Chem. 4, 141 (1968).

${ }^{16}$ G. W. F. Drake, H. Doyle, and A. Dalgarno (unpublished).

${ }^{17}$ W. Perl and V. Hughes, Phys. Rev. 91, 842 (1953). 